\title{
Yagi-Uda Antenna Design Optimization for Maximum Gain using different BBO Migration Variants
}

\author{
Satvir Singh \\ SBS State Technical Campus \\ Ferozepur, Punjab [INDIA]
}

\author{
Gagan Sachdeva \\ SBS State Technical Campus \\ Ferozepur, Punjab [INDIA]
}

\begin{abstract}
Biogeography is the study of distribution of biological species, over space and time, among random habitats. Recently developed Biogeography-Based Optimization (BBO) is a technique, where solutions of the problem under consideration are named as habitats; similar to chromosome in Genetic Algorithms (GAs) and particles in Particle Swarm Optimization (PSO). Feature sharing among various habitats, i.e., exploitation, is made to occur due to migration operator wheras exploration of new SIV values, similar to that of GAs, is accomplished with mutation operator. In this paper, various migration variants of BBO algorithm, reported till date, are investigated to optimize the lengths and spacings for YagiUda antenna elements for maximum gain. The results obtained with these migration variants are compared and the best results are presented in the ending sections of the paper.
\end{abstract}

\section{Keywords:}

Yagi-Uda Antenna, Bio-geography Based Optimization (BBO), Migration Variants, Enhanced BBO, Immigration Refusal BBO, Blended BBOifx

\section{INTRODUCTION}

Antenna is an electrical device which forms an interface between free-space radiations and transmitter or receiver. The choice of an antenna depends on various factors such as gain, impedance, bandwidth, frequency of operation, Side Lobe Level (SLL), etc. A Yagi-Uda antenna is a widely used antenna design at VHF and UHF due to its high forward gain capability (typically, greater than $10 \mathrm{~dB}$ ), low cost and ease of construction. It is a parasitic linear array of parallel dipoles, one of which is energized directly by transmission line while others act as a parasitic radiators whose currents are induced by mutual coupling. The characteristics of Yagi-Uda antenna are affected by its geometric parameters.

A Yagi-Uda antenna was invented in 1926 by H. Yagi and S. Uda at Tohoku University [28] in Japan, however, published in English in 1928 [32]. Since then, continuous efforts have been put in improving the antenna design for higher gain, requisite impedance, minimum SLL and wider bandwidth using different optimization techniques based on traditional mathematical approaches [21, 3, 7, 22, 6, 5, 8] and modern heuristics of Artificial Intelligence (AI) [13, 31, 30, 2, 15, 27, 26].

In 1949, Fishenden and Wiblin [12] proposed an approximate design of Yagi aerials for maximum gain. In 1959, Ehrenspeck and Poehler have given a manual approach to maximize the gain of the antenna by varying various lengths and spacings of its elements [11]. Later, with the availability of computer software at affordable prices made it possible to optimize antennas, numerically. Bojsen et al. [3] proposed another optimization technique to maximize the gain of Yagi-Uda antenna arrays with equal and unequal spacings between adjoining elements. Cheng et al. [6] 5] have used optimum spacings and lengths to optimize the gain of a Yagi-Uda antenna. In [8], Cheng has proposed optimum design of the antenna where antenna gain function is highly non-linear.

In 1975, John Holland introduced Genetic Algorithms (GAs) as a stochastic swarm based AI technique, inspired from Darwinian theory of Survival of the fittest, to optimize arbitrary systems for certain fitness measurement function. Many researchers, since then, investigated GAs to optimize Yagi-Uda antenna designs for various objectives one-by-one [1, 13, 9] and collectively [31, 29, 14, 24]. Baskar et al., in [2], have optimized the antenna using Comprehensive Learning PSO (CLPSO) and presented better results than that of previously reported techniques. $\mathrm{Li}$ in [15] has used Differential Evolution (DE) to optimize geometrical parameters of a Yagi-Uda antenna and illustrated capabilities of the proposed method with several antenna designs. In [27], Singh et al. have explored another useful, stochastic global search and optimization technique named as Simulated Annealing (SA) for optimal Yagi-Uda antenna design.

In 2008, Dan Simon introduced yet another swarm based stochastic optimization technique, named BBO [23], inspired from the science of biogeography. Here, features sharing among various habitats, i.e., potential solutions, is accomplished with migration operator and exploration of new features is done with mutation operator. Singh et al. have presented BBO as a better alternative for optimizing Yagi-Uda antenna designs [26].

In [10], Du et al. have proposed the concept of immigration refusal in BBO aiming at improved performance. In [16], Ma and Simon introduced another migration operator, i.e., Blended migration, to solve constrained optimization problems and make BBO convergence faster. In [19], Pattnaik et al. have proposed Enhanced Biogeography-Based Optimization (EBBO) in which duplicate habitats, created due to migration of features, is replaced with randomly generated habitats to increase the exploitation ability of BBO algorithm.

In this paper, various migration variants in $\mathrm{BBO}$ algorithm are experimented to optimize the wire-lengths of Yagi-Uda antenna elements and their spacings to investigate relative optimization performance. A method of moments based programme, NEC2 (Numerical Electromagnetics Code version 2), is used to evaluate the antenna designs for gain, input impedance, bandwidth and beamwidth, etc.

After this brief literature review, the paper is outlined as follows: In the Section 2, Yagi-Uda antenna design parameters are discussed. Section 3 is dedicated to BBO algorithm and its variants. In Section 4, comparative simulation results obtained with different migration operators, during optimizing antenna designs, are presented. Finally, drawn conclusions and future scope have been discussed in Section 5 .

\section{THE ANTENNA DESIGN PARAMETERS}

Yagi-Uda antenna consists of three types of elements: (a) Reflector-biggest among all and is responsible for blocking radiations 
in one direction. (b) Feeder-which is fed with the signal from transmission line to be transmitted and (c) Directors-these are usually more then one in number and responsible of unidirectional radiations. Figure 1 depicts a typical six-element Yagi-Uda wire antenna with four directors parallel to $x$-axis and placed on $y$-axis. An incoming field sets up resonant currents on all the antenna elements which re-radiate signals. These re-radiated fields are then picked up by the feeder element, that leads to total current induced in the feeder equivalent to combination of the direct field input and re-radiated contributions from the director and reflector elements.

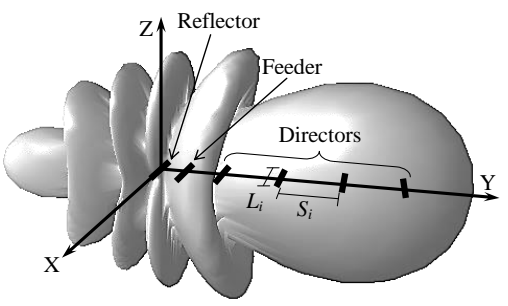

Fig. 1. Six-element Yagi-Uda Antenna

Designing a Yagi-Uda antenna involves determination of wirelengths and wire-spacings in between to get maximum gain, desired impedance and minimum SLL at a frequency of operation. Typically, an antenna with $N$ elements requires $M=2 N-1$ parameters, i.e., $N$ wire-lengths and $N-1$ their spacings, that are to be determined. These $M$ parameters, collectively, are represented as a string referred as a habitat, $H$, given as (1) or (2).

$$
H=\left[L_{1}, L_{2}, \ldots, L_{N}, S_{1}, S_{2}, \ldots, S_{N-1}\right]
$$

or

$$
H=\left[L_{1}, S_{1}, \ldots, L_{i}, S_{i}, L_{i+1}, \ldots, S_{N-1}, L_{N},\right]
$$

where $L_{i}$ is the length of $i$ th element and $S_{i}$ is the spacing between the $i$ th and $(i+1)$ th element.

\section{BIOGEOGRAPHY-BASED OPTIMIZATION}

As name suggests, $\mathrm{BBO}$ is a population based global optimization technique developed on the basis of the science of biogeography, i.e., study of the distribution of animals and plants among different habitats over time and space. BBO results presented by researchers are better than other EAs [13, 29, 2, 20].

Mathematical models of biogeography describe migration, speciation, and extinction of species in various islands [17]. The term island is used for any habitat that is geographically isolated from other habitats. Habitats that are well suited residences for biological species are referred to have high Habitat Suitability Index (HSI) similar to fitness in other EAs. However, HSI value of a habitat depends upon many factors such as rainfall, diversity of vegetation, diversity of topographic features, land area, and temperature, etc. These variables that characterize habitability are termed as Suitability Index Variables (SIVs). The habitats with a high HSI tend to have a large population of its resident species, that is responsible for more probability of emigration and less probability of immigration due to natural random behavior of species. Immigration is the arrival of new species into a habitat or population, while emigration is the act of leaving one's native region.

On the other hand, habitats with low HSI tend to have low emigration rate, $\mu$, due to sparse population, however, they will have high immigration rate, $\lambda$. Suitability of habitats with low HSI is likely to increase with influx of species from other habitats having high HSI. However, if HSI does not increase and remains low, species in that habitat go extinct that leads to additional immigration. For sake of simplicity, it is safe to assume a linear relationship between emigration rates and immigration rates. Additionally, same maximum emigration and immigration rates, i.e., $E=I$, as depicted graphically in Figure 2. For HSI of $k$ -

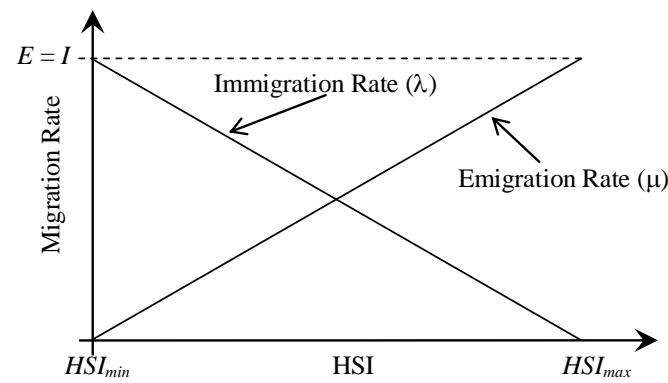

Fig. 2. Migration Curves

th habitat emigration and immigration rates are given by (3) and (4).

$$
\begin{gathered}
\mu_{k}=E \cdot \frac{H S I_{k}}{H S I_{\max }-H S I_{\min }} \\
\lambda_{k}=I \cdot\left(1-\frac{H S I_{k}}{H S I_{\max }-H S I_{\min }}\right)
\end{gathered}
$$

The immigration of new species from high HSI to low HSI habitats may raise the HSI of poor habitats as good solutions have more resistance to change than poor solutions whereas poor solutions are more dynamic and accept a lot of new features from good solutions. Following subsections discuss two constituents operators of $\mathrm{BBO}$ algorithm, viz. migration and mutation.

\subsection{Migration}

Migration is a probabilistic operator that improves HSI of poor habitats by sharing features from good habitats. During Migration, immigrating habitat, ImHbt, use its immigration rate, $\lambda$, given by (4), to probabilistically decide whether to immigrate or not. In case immigration is selected, then the emigrating habitat, EmHbt, is found probabilistically based on emigration rate, $\mu$, given by (3). The process of migration is completed by copying values of SIVs from EmHbt to ImHbt at random chosen sites. This is termed as the standard migration operator, in this paper. The migration operator may lead to same types of habitats in large number after few iterations. In this paper, different migration variants are investigated to increase the diversity in the population, with objective of improved performance BBO algorithm whose pseudo code are depicted in Algorithm 1 and discussed in the following subsections.

3.1.1 Immigration Refusal. In BBO, if a habitat has high emigration rate, i.e, the probability of emigrating to other habitats is high and the probability of immigration from other habitats is low. However, the low probability does not mean that immigration will never happen. Once in a while, a highly fit solution may receive solution features from a low-fit solution that may degrade its fitness. In such cases, immigration is refused to prevent degradation of HSI values of habitats. This BBO variants with conditional migration is termed as Immigration Refusal BBO [10] whose performance with testbed of benchmark functions is encouraging. 
3.1.2 Blended Migration. Blended migration operator [16] is a generalization of the standard $\mathrm{BBO}$ migration operator that has got inspiration from blended crossover in GAs [18]. Here, SIVs of immigrating habitat, ImHbt, is not simply replaced by SIVs of emigrating habitat, $\mathrm{EmHbt}$, like standard migration operation. Rather, a new value for $i$-th SIV, $S I V_{\text {new }}$, is comprised of two components given as (5)

$$
S I V_{\text {new }}=\alpha \cdot \operatorname{ImHbt}(S I V)+(1-\alpha) \cdot \operatorname{EmHbt}(S I V)
$$

where $\alpha$ is a random number between 0 and 1 . The pseudo code of blended migration is depicted as case 3 in Algorithm 1.

3.1.3 Enhanced Biogeography Based Optimization. Standard BBO migration operator tends to create duplicate solutions which decreases the diversity in the population. To prevent this diversity decrease, duplicate habitats are replaced with randomly generated habitats, as depicted as case 4 in Algorithm 1. This leads to increase exploration of new SIV values. In EBBO, clear duplicate operator is integrated in basic $\mathrm{BBO}$ to improve its performance [19].

\subsection{Mutation}

Mutation is probabilistic operator that randomly modifies the values of some randomly selected SIVs. It is intended for exploration of search space for better SIV values by increasing the diversity in the population. Here, increased mutation rate is investigated on habitats those are, probabilistically, participating less in migration. The mutation rate, mRate, for $i$-th habitats is determined as (6)

$$
\text { mRate }=C \cdot \min \left(\mu_{k}, \lambda_{k}\right)
$$

where $C$ is a constant and equal to 3 [25]. The pseudo code for standard mutation operator is depicted in Algorithm 2. In our simulations, like other population-based optimization techniques, elitism is considered so as to preserve best solution throughout the process of optimization.

\section{SIMULATION RESULTS AND DISCUSSIONS}

Six-wire Yagi-Uda antenna designs are optimized for gain using BBO where all migration variants, discussed in Section 3.1, are investigated along with standard mutation operator. Average of 10 monte-carlo evolutionary runs for each migration operators are presented here for fair comparative study of stochastic algorithm variants, viz. immigration refusal, blended migration, EBBO and standard migration, as depicted in Figure 3. The C++ programming platform is used for coding of $\mathrm{BBO}$ algorithm,

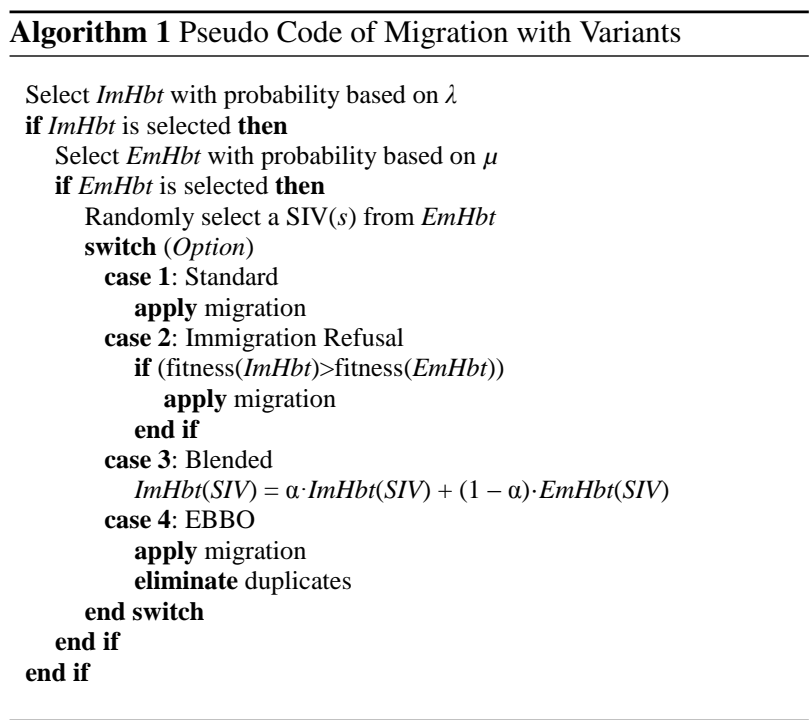

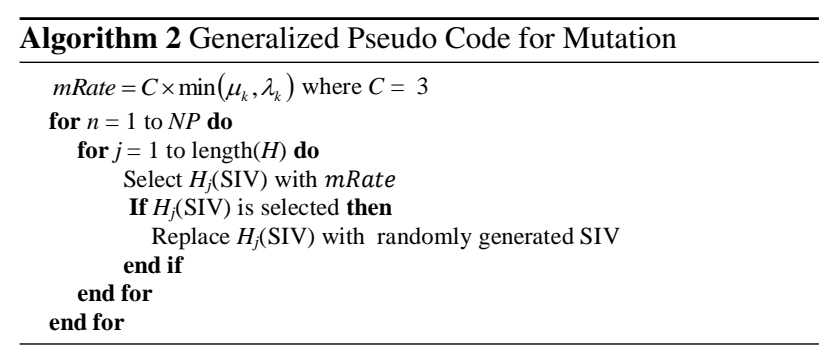

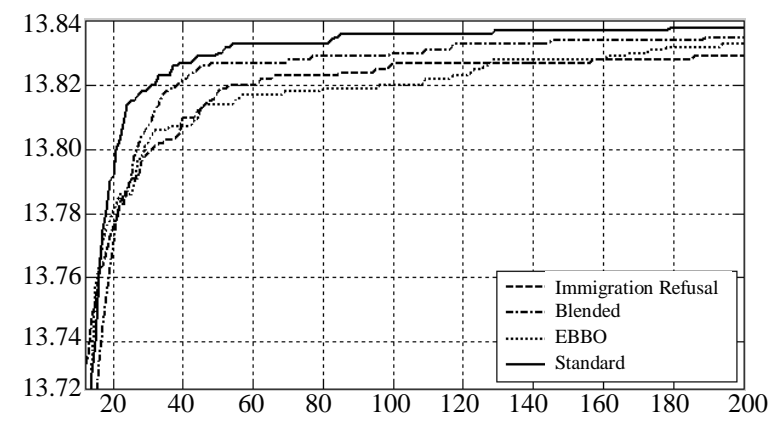

Fig. 3. Convergence Performance of BBO with Migration Options

\begin{tabular}{|c|c|c|c|c|c|c|c|c|}
\hline & \multicolumn{2}{|c|}{ Standard } & \multicolumn{2}{|c|}{ Immi. Refusal } & \multicolumn{2}{|c|}{ Blended } & \multicolumn{2}{|c|}{ EBBO } \\
\hline Element & Length & Spacing & Length & Spacing & Length & Spacing & Length & Spacing \\
\hline $1(\lambda)$ & 0.4832 & & 0.4845 & & 0.4838 & & 0.4853 & \\
\hline $2(\lambda)$ & 0.4794 & 0.201 & 4927 & 180 & 4689 & 1695 & 0.4888 & 1527 \\
\hline $3(\lambda)$ & 0.4410 & 0.2347 & 0.4398 & 0.2439 & 4414 & 0.2414 & 0.4411 & 0.2523 \\
\hline $4(\lambda)$ & 0.42 & 11 & 0.4234 & 4 & 0.4265 & 0.3753 & 0.4268 & 0.3814 \\
\hline $5(\lambda)$ & 0.4205 & 0.4205 & 0.4201 & 0.4164 & \begin{tabular}{|l|}
0.4181 \\
\end{tabular} & 0.4417 & 0.4185 & 0.4231 \\
\hline $6(\lambda)$ & 0.4294 & 0.3824 & 0.4256 & 0.3878 & \begin{tabular}{|l|}
0.4274 \\
\end{tabular} & 0.3762 & 0.4236 & 0.3906 \\
\hline Gain(dB & \multicolumn{2}{|c|}{13.84} & \multicolumn{2}{|c|}{13.84} & \multicolumn{2}{|c|}{13.84} & \multicolumn{2}{|c|}{13.84} \\
\hline $\mathrm{Z}(\boldsymbol{\Omega})$ & \multicolumn{2}{|c|}{$4.78+j 42.84$} & \multicolumn{2}{|c|}{$5.62+\mathrm{j} 64.60$} & \multicolumn{2}{|c|}{$3.58+\mathrm{j} 24.68$} & \multicolumn{2}{|c|}{$3.68+j 54.11$} \\
\hline
\end{tabular}

Table 1. The best results obtained during gain optimization

whereas, a NEC2 [4] is used for evaluation of antenna designs based on method of moments. Each potential solution in BBO is encoded as vector with 11 SIVs as given by (0??). The universe of discourse for the search of optimum values of wire-lengths and wire-spacings are $0.40 \lambda-0.50 \lambda$ and $0.10 \lambda-0.45 \lambda$, respectively, however, cross sectional radius and segment sizes are kept same for all elements, i.e., $0.003397 \lambda$ and $0.1 \lambda$ respectively, where $\lambda$ is the wavelength corresponding to frequency of operation, i.e, $300 \mathrm{MHz}$. Excitation is applied to the middle segment of driven element and location of middle segment of the reflector element is always kept at $x=0$. Typically, the best antenna designs obtained during process of optimization are tabulated in Table 1 .

EBBO algorithm yields poorest solutions in initial iterations and later improves significantly than immigration refusal variant. Blended migration variant gives mediocre performance. Comparatively, standard migration option gives the best convergence performance during optimizing Yagi-Uda antenna design for maximum gain in limited 200 iterations.

In our application of optimizing Yagi-Uda antenna design standard migration operator yields the best performance in limited 200 iterations. However, if run for large number of iterations EBBO may turn to be better option.

\section{CONCLUSIONS AND FUTURE SCOPE}

In this paper, BBO algorithm is applied to optimize six-element Yagi-Uda antenna designs for gain maximization. Here, BBO is experimented with different migration variants, viz., (1) Immigration Refusal, (2) Blended migration, (3) EBBO and (4) Stan- 
dard migration, along with standard mutation operator. Here, it is observed that standard migration operator is the best option for optimizing the antenna designs. Investigation of BBO algorithms for other types of antenna designs is next on our agenda.

\section{REFERENCES}

[1] E.E. Altshuler and D.S. Linden. Wire-antenna Designs using Genetic Algorithms. Antennas and Propagation Magazine, IEEE, 39(2):33-43, 1997.

[2] S. Baskar, A. Alphones, P N Suganthan, and J J Liang. Design of Yagi-Uda Antennas using Comprehensive Learning Particle Swarm Optimisation. IEEE, 152(5):340-346, 2005.

[3] JH Bojsen, H. Schjaer-Jacobsen, E. Nilsson, and J. Bach Andersen. Maximum Gain of Yagi-Uda Arrays. Electronics Letters, 7(18):531-532, 1971.

[4] G. J. Burke and A. J. Poggio. Numerical Electromagnetics Code (NEC) method of moments. NOSC Tech. DocLawrence Livermore National Laboratory, Livermore, Calif, USA, 116:1-131, 1981

[5] C. Chen and D. Cheng. Optimum Element Lengths for Yagi-Uda Arrays. IEEE Transactions on Antennas and Propagation, 23(1):8-15, 1975.

[6] D. Cheng and C. Chen. Optimum Element Spacings for Yagi-Uda Arrays. IEEE Transactions on Antennas and Propagation, , 21(5):615-623, 1973.

[7] D. K. Cheng. Optimization Techniques for Antenna Arrays. Proceedings of the IEEE, 59(12):1664-1674, 1971.

[8] D. K. Cheng. Gain Optimization for Yagi-Uda Arrays. Antennas and Propagation Magazine, IEEE, 33(3):42-46, 1991.

[9] D. Correia, A. J. M. Soares, and M. A. B. Terada. Optimization of gain, impedance and bandwidth in Yagi-Uda Antennas using Genetic Algorithm. IEEE, 1:41-44, 1999.

[10] D. Du, D. Simon, and M. Ergezer. Biogeography-based Optimization Combined with Evolutionary Strategy and Immigration Refusal. IEEE, 1:997-1002, 2009.

[11] H. Ehrenspeck and H. Poehler. A New Method for Obtaining Maximum Gain from Yagi Antennas. IRE Transactions on Antennas and Propagation, , 7(4):379-386, 1959.

[12] R. M. Fishenden and E. R. Wiblin. Design of Yagi Aerials. Proceedings of the IEE-Part III: Radio and Communication Engineering, 96(39):5, 1949.

[13] E. A. Jones and W. T. Joines. Design of Yagi-Uda Antennas using Genetic Algorithms. IEEE Transactions on Antennas and Propagation, , 45(9):1386-1392, 1997.

[14] Y. Kuwahara. Multiobjective Optimization Design of YagiUda Antenna. IEEE Transactions on Antennas and Propagation, 53(6):1984-1992, 2005.

[15] J. Y. Li. Optimizing Design of Antenna using Differential Evolution. IEEE, 1:1-4, 2007.

[16] H. Ma and D. Simon. Blended Biogeography-based Optimization for Constrained Optimization. Engineering Applications of Artificial Intelligence, 24(3):517-525, 2011.

[17] R.H. MacArthur and E.O. Wilson. The Theory of Island Biogeography. Princeton Univ Pr, 1967.

[18] T. McTavish and D. Restrepo. Evolving Solutions: The Genetic Algorithm and Evolution Strategies for Finding Optimal Parameters. Applications of Computational Intelligence in Biology, 1:55-78, 2008.

[19] S. S. Pattnaik, M. R. Lohokare, and S. Devi. Enhanced Biogeography-Based Optimization using Modified Clear Duplicate Operator. IEEE, 1:715-720, 2010.
[20] M. Rattan, M. S. Patterh, and B. S. Sohi. Optimization of Yagi-Uda Antenna using Simulated Annealing. Journal of Electromagnetic Waves and Applications, 22, 2(3):291299, 2008.

[21] D. G. Reid. The Gain of an Idealized Yagi Array. Journal of the Institution of Electrical Engineers-Part IIIA: Radiolocation, , 93(3):564-566, 1946.

[22] L. C. Shen. Directivity and Bandwidth of Single-band and Double-band Yagi Arrays. IEEE Transactions on Antennas and Propagation, 20(6):778-780, 1972.

[23] D. Simon. Biogeography-based Optimization. IEEE Transactions on Evolutionary Computation,, 12(6):702-713, 2008.

[24] Satvir Singh, Eitika Mittal, and Gagan Sachdeva. MultiObjective Gain-Impedance Optimization of Yagi-Uda Antenna using NSBBO and NSPSO. International Journal of Computer Applications, 56(15):1-6, 2012.

[25] Satvir Singh and Gagan Sachdeva. Mutation Effects on BBO Evolution in Optimizing Yagi-Uda Antenna Design. In proceeding of IEEE, 2012.

[26] U. Singh, H. Kumar, and T. S. Kamal. Design of Yagi-Uda Antenna Using Biogeography Based Optimization. IEEE Transactions on Antennas and Propagation, 58(10):33753379, 2010.

[27] U. Singh, M. Rattan, N. Singh, and M. S. Patterh. Design of a Yagi-Uda Antenna by Simulated Annealing for Gain, Impedance and FBR. IEEE, 1:974-979, 2007.

[28] Shintaro Uda and Yasuto Mushiake. Yagi-Uda Antenna. Maruzen Company, Ltd, 1954

[29] N. V. Venkatarayalu and T. Ray. Single and Multi-Objective Design of Yagi-Uda Antennas using Computational Intelligence. IEEE, 2:1237-1242, 2003.

[30] N.V. Venkatarayalu and T. Ray. Optimum Design of YagiUda Antennas Using Computational Intelligence. IEEE Transactions on Antennas and Propagation,, 52(7):18111818, 2004

[31] H. J. Wang, K. F. Man, C. H. Chan, and K. M. Luk. Optimization of Yagi array by Hierarchical Genetic Algorithms. IEEE, 1:91-94, 2003.

[32] H. Yagi. Beam Transmission of Ultra Short Waves. Proceedings of the Institute of Radio Engineers, 16(6):715$740,1928$.

Satvir Singh was born on Dec 7, 1975. He received his Bachelor's degree (B.Tech.) from Dr. B. R. Ambedkar National Institute of Technology, Jalandhar, Punjab (India) with specialization in Electronics \& Communication Engineering in year 1998, Masters degree (M.E.) from Delhi Technological University (Formerly, Delhi College of Engineering), Delhi (India) with distinction in Electronics \& Communication Engineering in year 2000 and Doctoral degree (Ph.D.) from Maharshi Dayanand University, Rohtak, Haryana (India) in year 2011.

During his 12 years of teaching experience he served as Assistant Professor and Head, Department of Electronics \& Communication Engineering at BRCM College of Engineering \& Technology, Bahal, (Bhiwani) Haryana, India and as Associate Professor \& Head, Department of Electronics \& Communication Engineering at Shaheed Bhagat Singh State Technical Campus (Formerly, SBS College of Engineering \& Technology), Ferozepur Punjab, India.

His fields of special interest include Evolutionary Algorithms, High Performance Computing, Type-1 \& Type-2 Fuzzy Logic Systems, Wireless Sensor Networks and Artificial Neural Networks for solving engineering problems. He is active member of 
an editorial board of International Journal of Electronics Engineering and published nearly 15 research papers in International Journals and Conferences. He has delivered nearly 15 Invited Talks during National and International Conferences, Seminar, Short Term Courses and Workshops. He completed two AICTE funded projects under MODROB Scheme worth 15 Lacs and conducted a 2-Week Staff Development Programme on "Intelligent Computational Techniques".

Gagan Sachdeva was born on Oct 4, 1988. He received his Bachelor's degree (B.Tech.) and Master's degree (M.Tech.) from
Shaheed Bhagat Singh State Technical Campus (formerly, SBS College of Engineering \& Technology), Ferozepur, Punjab (India) with specialization in Electronics \& Communication Engineering in year 2010 and 2012, respectively. He is presently with Shaheed Bhagat Singh State Technical Campus, Ferozepur as Adhoc Lecturer in the department of ECE. His research interests include Evolutionary Algorithms, Antenna design optimization, and Wireless Sensor Networks. 